\title{
REMOÇÃO DE FLUIDO DE PERFURAÇÃO BASE ÓLEO POR COLCHÕES LAVADORES COMPOSTOS POR ÓLEO VEGETAL, TENSOATIVO E SALMOURA
}

\author{
F. D. S. Curbelo ${ }^{1}$, R. M. Aranha ${ }^{1}$, E. A. Araújo ${ }^{1}$, J. C. O. Freitas ${ }^{2}$, A. I. C. Garnica ${ }^{1}$ \\ ${ }^{1}$ Universidade Federal da Paraíba, ${ }^{2}$ Universidade Federal do Rio Grande do Norte \\ fabioladias@yahoo.com*
}

Submetido 08/02/2017 - Aceito 26/06/2017

DOI: $10.15628 /$ holos.2017.5631

\section{RESUMO}

Os fluidos de perfuração base óleo têm sido utilizados em perfuração de poços de petróleo por apresentarem características como, lubricidade, fricção mecânica, inibição química, estabilidade do sistema, entre outras, pois minimizam inúmeros problemas durante a etapa de perfuração. Porém, o reboco formado pelo fluido de perfuração nas paredes do poço e o fluido contido em seu interior devem ser removidos de forma eficiente, propiciando uma boa cimentação, melhorando a aderência do cimento a formação e do cimento ao revestimento. Os colchões lavadores são fluidos que possuem propriedades químicas adequadas que atuam dispersando o reboco e o fluido de perfuração. Baseado nisto, este trabalho teve o objetivo de desenvolver
\end{abstract}

colchões lavadores a base de óleo vegetal e tensoativos, para serem utilizados durante a operação de cimentação de poços de petróleo removendo o reboco formado pelo fluido de perfuração nas paredes do poço. Foram utilizados três tensoativos nas formulações dos colchões, um aniônico de origem vegetal (OCS) e dois não iônicos (TW80 e ASP80). E suas eficiências foram avaliadas a partir de testes de remoção. Dos resultados obtidos, verificou-se que os tensoativos OCS e ASP80 apresentaram valores satisfatórios de remoção de aproximadamente $77 \%$ e $97 \%$, respectivamente. Sendo o OCS uma boa alternativa para uso em formulações de colchões lavadores, uma vez que este é um tensoativo de origem vegetal.

PALAVRAS-CHAVE: óleo vegetal, tensoativo, colchão lavador, fluido de perfuração, remoção.

\section{Removal of oil based drilling fluid by preflushes composed of vegetable oil, surfactant and brine}

\begin{abstract}
Oil-based drilling fluids have been used in drilling oil wells because they present characteristics such as lubricity, mechanical friction, chemical inhibition, system stability, among others, as they minimize numerous problems during the drilling. However, thickness formed by the drilling fluid in the well and the fluid contained therein should be efficiently removed, providing a good cementation, improving the adhesion of the cement to formation and the cement to the pipe. Preflushes are fluids that have adequate chemical properties that act by dispersing the drilling fluid thickness and the drilling fluid. Based on this, the objective of this work was to develop preflushes based on vegetable oil and
\end{abstract}

surfactants, to be used during the cementing operation of oil wells by removing the formed thickness in well. Three surfactants were used in the formulations of the preflushes, an anionic of vegetable origin (OCS) and two nonionic (TW80 and ASP80). And their efficiencies were evaluated from the removal tests. From the results obtained, it was verified that the OCS and ASP80 surfactants had satisfactory removal values of approximately $77 \%$ and $97 \%$, respectively. Being OCS a good alternative for use in flushes formulations, since this is a surfactant of vegetable origin.

KEYWORDS: vegetable oil, surfactant, preflushes, drilling fluid, removal. 


\section{INTRODUÇÃO}

Nos últimos anos, poços de petróleo têm sido perfurados em cenários cada vez mais complexos, com estreita janela operacional, lâminas d’água profundas, entre outras (Quintero et at., 2015). Nesses locais, fluidos de perfuração base óleo têm sido utilizados por apresentarem características como, lubricidade, friç̧ão mecânica, inibição química, estabilidade do sistema, entre outras, superiores quando comparadas com os fluidos de perfuração base água, minimizando a ocorrência de inúmeros problemas durante a etapa de perfuração (Osode et al., 2012).

Segundo See et al. (2011), os fluidos de perfuração ainda fornecem pressão hidrostática sobre a formação, impedindo que haja migração dos fluidos da formação para o interior do poço, minimizam o atrito entre a coluna e as paredes do poço, e formam um reboco fino e de baixa permeabilidade evitando que ocorra perda de fluido de perfuração para a formação, entre outras funções.

Brandl et al. (2013) mencionaram que, após a conclusão da fase de perfuração, o reboco formado nas paredes do poço e o fluido contido em seu interior devem ser removidos de forma eficiente, propiciando condições adequadas para o assentamento do revestimento e na mitigação de possíveis danos a formação. De acordo com Pernites et al. (2015), a remoção ineficiente desses parâmetros (reboco e fluido contido no interior do poço) poderá afetar diretamente a qualidade da cimentação, colocando em risco a integridade do poço por falta de vedação hidráulica ao revestimento, gerando comunicação entre zonas, podendo inclusive levar a ocorrência de kicks e blowouts.

Segundo Quintero et al. (2015), a remoção eficiente desse material se tornou um dos grandes desafios do setor petrolífero devido à dissimilaridade química existente entre os fluidos de perfuração base óleo e as pastas de cimento que, quando entram em contato, formam uma mistura viscosa, diminuindo a eficiência de remoção do reboco formado nas paredes do poço.

Para aumentar a eficiência da remoção do reboco, na etapa de cimentação de poços de petróleo, é importante a aplicação de fluidos com características especiais, denominados de colchão, bombeados à frente da pasta de cimento (Berry, 2005). Esses colchões devem ser cuidadosamente concebidos para evitar a interação com os fluidos de perfuração e a pasta de cimento, propiciando a remoção eficiente do reboco formado nas paredes do poço, melhorando a aderência do cimento a formação e do cimento ao revestimento (Brandl et al., 2013).

Berry (2005) classifica esses sistemas como colchões lavadores por possuírem propriedades químicas que atuam dispersando o reboco e o fluido de perfuração. Pinheiro (2013) menciona que esses colchões podem ser constituídos à base de água ou óleo, tensoativos e dispersantes, com a função de afinar e dispersar o fluido de perfuração contido nas paredes da formação. Os tensoativos, com porções hidrofóbicas e hidrofílicas, são adicionados aos colchões com a finalidade de corrigir a molhabilidade da formação e na diluição da fase oleosa (Quintero et al., 2012).

Diante desse cenário, este trabalho teve como objetivo desenvolver colchões lavadores a base de óleo vegetal e tensoativos, para serem utilizados durante a operação de cimentação de 
poços de petróleo, com o objetivo de remover o reboco formado pelo fluido de perfuração nas paredes do poço, acarretando numa cimentação eficiente do revestimento.

\section{ASPECTOS TEÓRICOS}

\subsection{Colchão lavador}

Para proporcionar uma remoção eficiente do fluido de perfuração no interior do poço, são bombeados colchões, lavadores e espaçadores, a frente da pasta de cimento, com funções distintas na operação de cimentação.

Os colchões lavadores têm função química no interior do poço, proporcionando a remoção do fluido de perfuração contido nas paredes e no interior do poço, melhorando a aderência do cimento a formação/revestimento. Os colchões espaçadores são fluidos viscosos, com determinado peso específico, que apresentam mecanismos físicos na operação, formando, com isso, uma barreira entre a pasta de cimento e o fluido de perfuração (Campos, 2009).

A Figura 1 mostra a atuação dos fluidos, colchões lavador e espaçador, durante a operação de cimentação de poços. Nessa operação, o colchão lavador, bombeado no interior da coluna, retorna a superfície pelo espaço anular, deslocando o fluido para superfície e removendo o reboco formado nas paredes do poço.

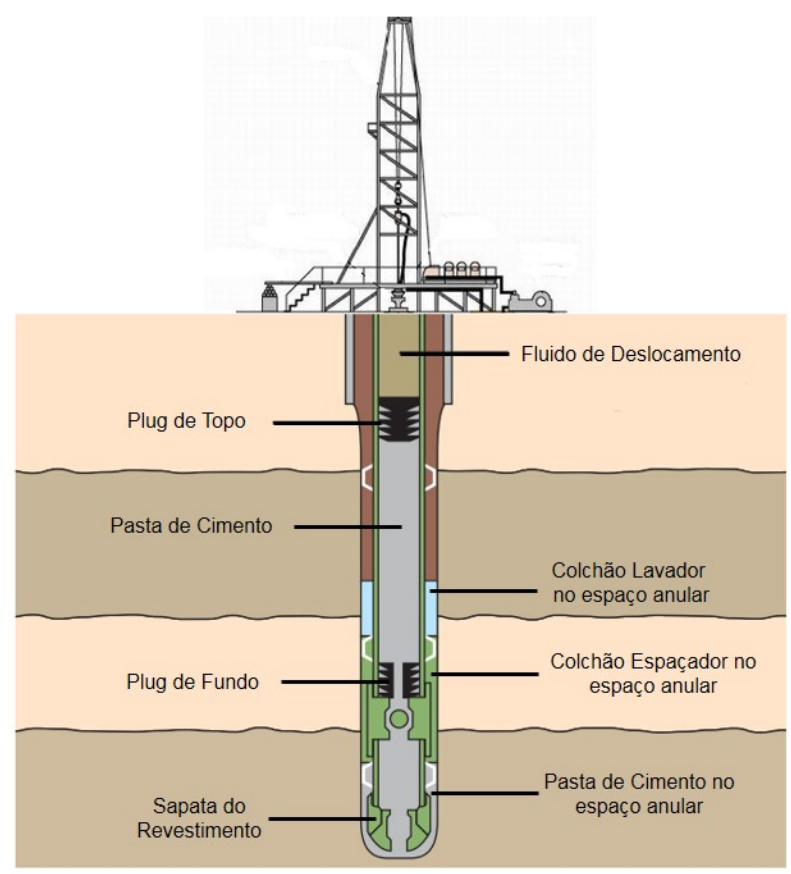

Figura 1: Esquema de bombeamento do colchão lavador no interior do poço.

Segundo Lima (2012), os seguintes aspectos devem ser considerados na formulação desses colchões:

- Remoção de todos os vestígios de fluido de perfuração;

- Preservação da integridade do fluido de perfuração removido;

- Substituição do fluido de perfuração pelo cimento; 
- Completar as tarefas com o mínimo de risco para os operadores e o meio ambiente;

- Baixo custo.

Com o intuito de aumentar a eficiência desses colchões em campo, têm sido utilizados tensoativos. Esses tensoativos tem a finalidade de otimizar o processo de remoção do fluido contido nas paredes do poço e a inversão da molhabilidade da parede do poço, consequentemente, reduzindo custos dos colchões, lavador e espaçador, garantindo a aderência da interface do tubo-cimento-formação.

\subsection{Tensoativos}

Os tensoativos (Figura 2), também conhecidos como surfactantes, são substâncias naturais ou sintéticas que possuem em sua estrutura química grupos polares (hidrofílicos), com afinidade pela fase aquosa, e grupos apolares (hidrofóbicos), com afinidade pela fase orgânica (Daltin, 2011).

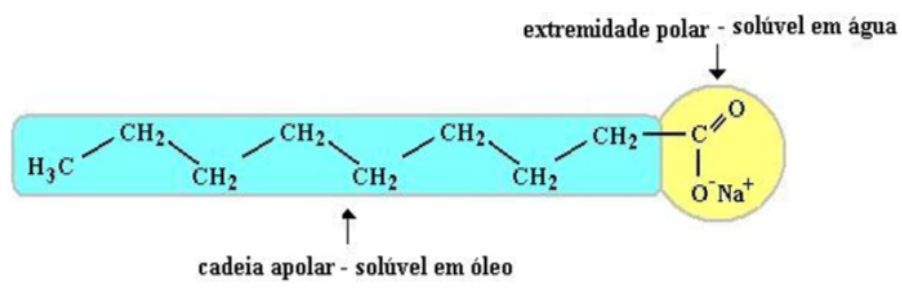

Figura 2: Molécula tensoativa com ambos os grupos (polares e apolares) (adaptado de Curbelo, 2006).

Segundo Daltin (2011), devido a dupla característica de afinidade na molécula, estes tendem a se concentrar na interface de um sistema, onde a molécula se orienta com a parte hidrofóbica voltada para substância de pouca afinidade com a água, e a parte hidrofílica voltada para a fase aquosa, formando um filme interfacial. Em altas concentrações, os tensoativos saturam a interface e, se encontram também, no seio da solução em forma de monômeros e micelas, como pode ser observado na Figura 3.

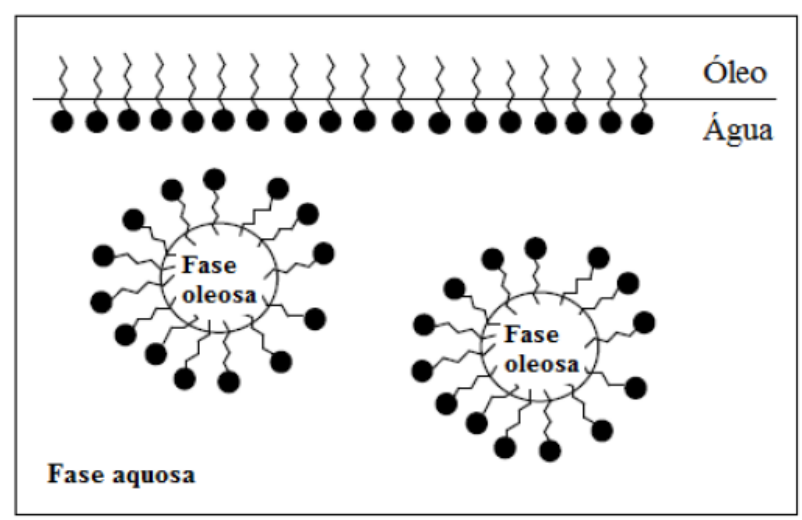

Figura 3: Adsorção de tensoativo na interface líquido-líquido e orientação das micelas (adaptado de Rossi, 2006).

Os tensoativos podem ser divididos em dois grupos, de acordo com a natureza da parte hidrofílica, em tensoativos iônicos e não iônicos. Os tensoativos iônicos podem ser classificados 
em função da carga elétrica do grupo hidrofílico presente na molécula em tensoativos aniônicos, catiônicos e anfóteros. Os tensoativos aniônicos, quando dissociados em água, acarretam a formação de íons carregados negativamente na superfície ativa do composto. Exemplos destes tensoativos são os sabões, os compostos sulfatados, sulfonados e fosfatados. Os tensoativos catiônicos, quando dissociados em meio aquoso, proporcionam a formação de íons carregados positivamente na superfície dos componentes, como por exemplo, o sal de amônio quaternário.

Os tensoativos anfóteros apresentam uma molécula de caráter duplamente iônico, com características aniônicas ou catiônicas, dependendo do $\mathrm{pH}$ do meio. Usualmente, para valores de $\mathrm{pH}$ inferiores a 4, tais tensoativos assumem características catiônicas. Para valores de pH entre 4 e 9 atuam como não-iônicos, e para valores entre 9 e 14 funcionam como tensoativos aniônicos.

Os tensoativos não iônicos apresentam-se como moléculas não dissociadas em solução aquosa e sua solubilidade média se deve à presença de grupos hidróxi ou polioxietilênicos contidos na estrutura (Attwood e Florence, 1983). O balanço hidrofílico lipofílico (BHL) dos tensoativos é um balanço quantitativo entre as características hidrofílicas e lipofílicas (hidrofóbicas) de uma molécula em solução, ou seja, é uma forma de quantificar os efeitos das contribuições das partes polar e apolar existentes na estrutura da molécula (Daltin, 2011). Esta escala foi introduzida para caracterizar os tensoativos não-iônicos utilizando, como grupo hidrofílico, o óxido de etileno.

A solubilidade do tensoativo não iônico varia de acordo com o $\mathrm{BHL}$ calculado para ele. Quanto maior o BHL, mais solúvel em água é o tensoativo. Os valores de BHL variam de 1 a 20 . Em que, o valor 20 corresponde a um percentual de $100 \%$ de caráter hidrofílico. O valor 01 corresponde a um percentual de $100 \%$ de caráter hidrofóbico. Um tensoativo com BHL igual a 10 tem as frações em peso das partes hidrofílicas (óxidos de etileno) e hidrofóbicas iguais, ou seja, tem igual afinidade tanto pelo o óleo quanto pela água.

\section{METODOLOGIA}

\subsection{Síntese do colchão lavador}

Para a elaboração dos colchões lavadores, algumas formulações foram determinadas (Tabela 1).

Tabela 1: Formulações dos colchões lavadores analisados.

\begin{tabular}{c|c|c|c}
\hline Formulação & Tensoativos & Relação $v_{\text {tens }}(\mathrm{mL}) / v_{\text {óleo }}(\mathrm{mL})$ & $C_{\text {tens }}(\mathrm{g} / \mathrm{L})$ \\
\hline 1 & OCS & $100 / 100$ & 12 \\
\hline 2 & OCS & $100 / 100$ & 30 \\
\hline 3 & OCS & $140 / 60$ & 12 \\
\hline 4 & OCS & $140 / 60$ & 30 \\
\hline 5 & TW80 & $100 / 100$ & 12 \\
\hline 6 & TW80 & $100 / 100$ & 30 \\
\hline 7 & TW80 & $140 / 60$ & 12 \\
\hline 8 & TW80 & $140 / 60$ & 30 \\
\hline 9 & ASP80 & $100 / 100$ & 12 \\
\hline 10 & ASP80 & $100 / 100$ & 30 \\
\hline 11 & ASP80 & $140 / 60$ & 12 \\
\hline
\end{tabular}




\begin{tabular}{l|l|l|l}
\hline 12 & ASP80 & $140 / 60$ & 30 \\
\hline
\end{tabular}

As formulações foram constituídas por: uma fase aquosa, solução salina de $2 \%$ em peso de $\mathrm{NaCl}$ ou salmoura; uma fase oleosa, óleo vegetal e um tensoativo. Três tensoativos foram utilizados nas formulações: óleo de coco saponificado (OCS), de origem vegetal; Tween 80 (TW80) e Alkest SP 80 (ASP80), obtidos comercialmente. Sendo o OCS um tensoativo aniônico e o T80 e ASP80 tensoativos não iônicos.

Os reagentes, solução aquosa de tensoativos com óleo vegetal, foram adicionados em recipiente adequado obtendo-se o denominado colchão lavador.

As formulações são diferenciadas pelo tipo tensoativo, pela relação volume da solução de tensoativo $(\mathrm{mL})$ /volume de óleo $(\mathrm{mL})$ e pela concentração de tensoativo utilizada na fase aquosa (C em g/L).

\subsection{Teste de eficiência de remoção}

O teste de remoção consiste em analisar, através de uma janela de visualização (Figura 4), o percentual removido de fluido de perfuração através do colchão lavador. Para simular as paredes do poço, uma janela de visualização é observada em uma área delimitada (de $6 \mathrm{~cm}$ de altura e $11 \mathrm{~cm}$ de comprimento) num béquer de $250 \mathrm{~mL}$. A área delimitada é confeccionada em material adesivo, transparente, com subdivisões de $1 \mathrm{~cm}$ por $1 \mathrm{~cm}$, com a finalidade de facilitar a visualização da área de fluido de perfuração removida pelo colchão lavador.

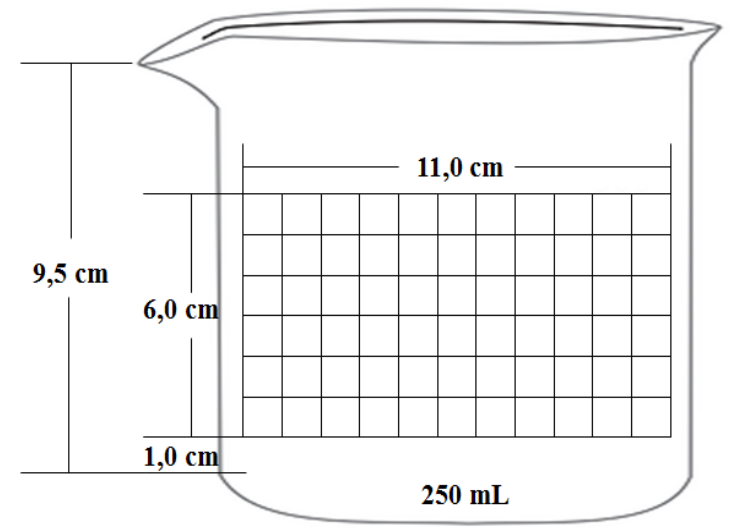

Figura 4: Representação esquemática da janela de visualização.

O fluido de perfuração não aquoso utilizado nas análises de remoção foi a base de nparafina, tendo sido utilizado em operações de perfuração de poços terrestres, no Estado do Rio Grande do Norte. As etapas para aplicação do fluido de perfuração na área delimitada do béquer e o teste de remoção estão mostrados a seguir (Figura 5):

- Homogeneizar o fluido de perfuração por 15 minutos no Hamilton Beach;

- Coletar $4 \mathrm{~mL}$ de fluido de perfuração com uma seringa;

- Formar um filme sobre a janela de visualização (até $4 \mathrm{~mL}$ ), no interior do béquer; 
- Adicionar $200 \mathrm{~mL}$ de colchão lavador formulado, mantendo o filme integro no interior do béquer;

- Posicionar o béquer no centro da plataforma do viscosímetro rotativo Fann (modelo 35A);

- Acionar a rotação de 300 rpm e acionar o cronômetro;

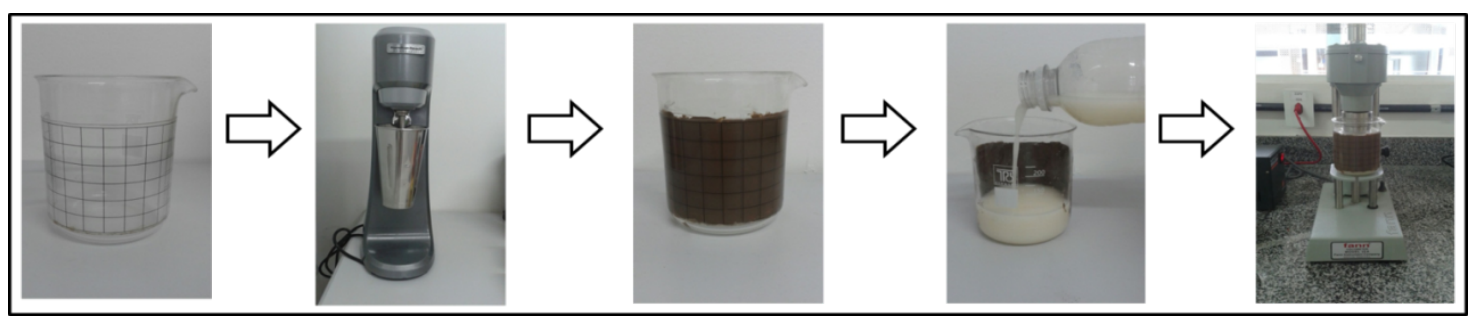

Figura 5: Etapas para o teste de eficiência de remoção do fluido de perfuração pelo colchão lavador.

O tempo do teste está limitado a 20 minutos, ou seja, se não houver remoção total do filme, o teste pode ser encerrado em 20 minutos, e se contabiliza o percentual da área removida.

A área de remoção de fluido de perfuração $\left(A_{\text {limpa }}\right)$ pode ser calculada pelo produto do número de quadrados limpos pela área do quadrado, conforme a Equação 1.

área de remoção $=n^{\circ}$ de quadrados removidos $x 1 \mathrm{~cm}^{2}$

O percentual de remoção pode ser obtido pela razão da área limpa (área de remoção) pela área total da janela de visualização, conforme a Equação 2.

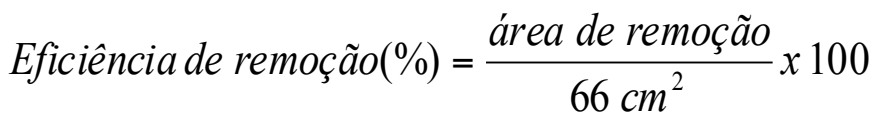

\section{RESULTADOS E DISCUSSÃO}

Dos resultados obtidos do teste de remoção, observa-se pela Figura 6 que o percentual de remoção variou de 1,5\% (formulação 7) até $97 \%$ (formulação 10), aproximadamente. 


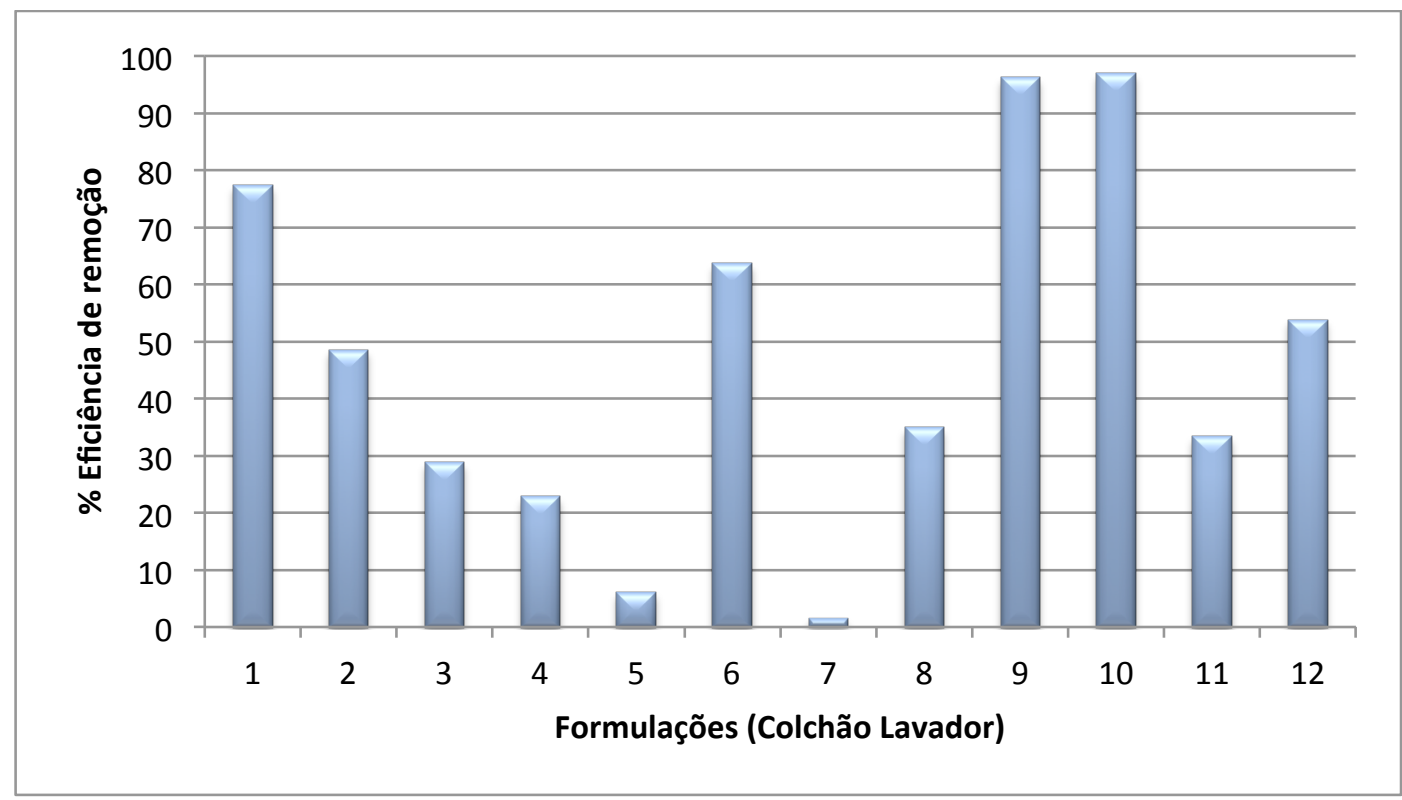

Figura 6: Percentual de eficiência de remoção do fluido de perfuração não aquoso através de colchões lavadores.

Dentre os tensoativos estudados, observou-se que as formulações com ASP80 apresentou os maiores percentuais de remoção, que foram de $96,21 \%$ e 96,97\%, para as amostras 9 e 10, respectivamente. Isto pode ser justificado pelo fato do tensoativo ASP80 apresentar uma cadeia orgânica maior possuindo, com isso, maior afinidade pelo fluido não aquoso, quando comparado com o OCS e o TW80. E, portanto, o BHL (Balanço Hidrofílico-Lipofílico) do ASP80, com valor igual a 4,3, é inferior ao do TW80 (BHL $=15,0)$, indicando uma característica mais hidrofóbica do ASP80. O OCS é bastante solúvel em água, apresentando características mais hidrofílicas e, por ser um tensoativo iônico (aniônico), não possui valores de BHL.

Para as relações volumétricas estudadas, $v_{\text {tens }}(\mathrm{mL}) / \mathrm{v}$ óleo $(\mathrm{mL})$, com valores de 100/100 e 140/60, as formulações 1 , 2, 5, 6, 9 e 10 representam colchões com proporção de $100 \mathrm{~mL}$ tens $/ 100$ $\mathrm{mL}_{\text {óleo, }}$ e as demais amostras são formulações com relação $140 \mathrm{~mL}_{\text {tens }} / 60 \mathrm{~mL}_{\text {óleo, }}$ como observado na Tabela 1. De forma geral, independentemente do tipo de tensoativo testado, observou-se que quanto maior a quantidade do óleo vegetal na formulação do colchão lavador, maior o percentual de remoção, com exceção do TW80 (formulação 5), devido a sua boa solubilidade em água $(B H L=15,0)$, tendo, com isso, menor eficiência na remoção do fluido de perfuração base óleo.

A Tabela 2 mostra o percentual de remoção para os tensoativos estudados. Com relação à concentração do tensoativo na fase aquosa (salmoura), observou-se que o aumento da concentração do tensoativo na solução, de 12 para $30 \mathrm{~g} / \mathrm{L}$, promoveu um aumento no percentual de remoção para os tensoativos TW80 e ASP80. O contrário ocorreu para o OCS, ou seja, o aumento da concentração ocasionou uma redução na remoção, devido, provavelmente, ao fato do OCS (tensoativo iônico) solubilizar mais facilmente a fase oleosa na fase aquosa com o aumento da sua quantidade na formulação, quando comparado aos outros dois tensoativos não iônicos.

Observa-se, também, com relação aos tensoativos não iônicos, que o aumento da concentração foi mais influente na remoção para o TW80, em que o seu percentual aumentou de 6,06\% para 63,64\%, com o aumento da concentração de 12 para $30 \mathrm{~g} / \mathrm{L}$, respectivamente. Para o ASP80, não foi observado alteração significativa no percentual de remoção. 
Tabela 2: Eficiência de remoção das formulações estudadas.

\begin{tabular}{c|c|c|c|c}
\hline Formulação & Tensoativos & $C(\mathrm{~g} / \mathrm{L})$ & Relação $v_{\text {tens }}(\mathrm{mL}) / \mathrm{v}_{\text {óleo }}(\mathrm{mL})$ & $\begin{array}{c}\text { Remoção } \\
(\%)\end{array}$ \\
\hline 1 & OCS & 12 & $100 / 100$ & 77,27 \\
\hline 2 & OCS & 30 & $100 / 100$ & 48,48 \\
\hline 3 & OCS & 12 & $140 / 60$ & 28,79 \\
\hline 4 & OCS & 30 & $140 / 60$ & 22,73 \\
\hline 5 & TW80 & 12 & $100 / 100$ & 6,06 \\
\hline 6 & TW80 & 30 & $100 / 100$ & 63,64 \\
\hline 7 & TW80 & 12 & $140 / 60$ & 1,51 \\
\hline 8 & TW80 & 30 & $140 / 60$ & 34,85 \\
\hline 9 & ASP80 & 12 & $100 / 100$ & 96,21 \\
\hline 10 & ASP80 & 30 & $100 / 100$ & 96,96 \\
\hline 11 & ASP80 & 12 & $140 / 60$ & 33,33 \\
\hline 12 & ASP80 & 30 & $140 / 60$ & 53,79 \\
\hline
\end{tabular}

De forma geral, dos percentuais de remoção obtidos, verifica-se que a presença do óleo vegetal pode ser determinante para formulações de colchões lavadores para se obter bons percentuais de remoção, uma vez que o aumento da quantidade de óleo na proporção volumétrica das formulações melhorou a remoção do fluido de perfuração base óleo.

O tensoativo não iônico TW80 foi testado, porém seus resultados não foram tão satisfatórios, obtendo apenas $63,64 \%$ de remoção para uma concentração alta de tensoativo de $30 \mathrm{~g} / \mathrm{L}$. Os percentuais de remoção obtidos para os tensoativos OCS e ASP80 indicaram valores satisfatórios de aproximadamente $77 \%$ e $97 \%$, respectivamente, com concentração de tensoativo de $12 \mathrm{~g} / \mathrm{L}$.

\section{CONCLUSÃO}

Neste trabalho, verificou-se que formulações de colchões lavadores com soluções salinas de tensoativos e óleo vegetal podem ser efetivas na remoção de fluidos de perfuração base óleo em poços de petróleo, baseado nos testes de remoção efetuados, atingindo percentuais de remoção de até $97 \%$, sendo a quantidade de óleo vegetal o parâmetro de maior influência na remoção.

Dos tensoativos estudados, o OCS (aniônico) e o ASP80 (não iônico) são os mais indicados para serem utilizados nas formulações dos colchões lavadores. Sendo o OCS uma boa alternativa, uma vez que este tensoativo é de origem vegetal e, portanto, menos agressivo ao meio ambiente.

\section{REFERÊNCIAS}

Attwood, D.; \& Florence, A. T. (1983). Surfactants Systems, 1a Ed., London: Chapman and Hall.

Berry, S. L. (2005). Optimization of synthetic-based and oil-based mud displacements with an emulsion-based displacement spacer system. SPE Annual Technical Conference and Exhibition held in Dallas, Texas, 9-12 October, USA. doi: 10.2118/05273-MS 
Brandl, A., Acorda, E., Ellis, T., \& Bray, W. (2013). Improving mud removal and simplifying challenging cement desing: a deepwater case history in the South china sea. International Petroleum Technology Conference held in Beijing, 26-28 March, China. doi: 10.2523/IPTC-16761MS

Campos, G. (2009). Procelab - Procedimentos e métodos de laboratório destinados à cimentação de poços de petróleo.

Curbelo, F. D. S. (2006). Recuperação avançada de petróleo utilizando tensoativos (Tese de Doutorado em Engenharia Química). Universidade Federal do Rio Grande do Norte, Natal, RN, Brasil.

Daltin, D. (2011). Tensoativos: química, propriedades e aplicações. São Paulo, Editora Blucher.

Lima, F. M. (2012). Desenvolvimento de sistemas de colchões para aplicação em poços perfurados com fluidos de perfuração não aquosos (Dissertação de Mestrado em Engenharia Química). Universidade Federal do Rio Grande do Norte, Natal, RN, Brasil.

Osode, P., Otaibi, M., Moqbil, K. B., Kilani, K., \& Azizi, E. (2012). Evaluation of nonreactive aqueous spacer fluids for oil-based mud displacement in open hole horizontal well. International Petroleum Exhibition \& Conference held in Abu Dhabi, 11-14 November, UAE, doi: 10.2118/161914-MS

Pernites, R., Khammar, M., \& Santra, A. (2015). Robust spacer for water and oil based mud. Western Regional Meeting held in Garden Grove, 27-30 April, USA, doi: 10.2118/174005-MS.

Pinheiro, F. S. H. T. (2013). Desenvolvimento de misturas ternárias para remoção de reboco de fluido de perfuração sintético (Tese de Doutorado em Engenharia Química). Universidade Federal do Rio Grande do Norte, Natal, RN, Brasil.

See, C. H., Saphanuchat, W., Nadarajan, S., \& Lim., C. N. (2011). NanoEmulsion for non-aqueous mud removal in wellbore. SPE/DGS Saudi Arabia Section Technical Symposium and Exhibition, 15 - 18 May, Saudi Arabia. doi: 10.2118/148088-MS

Quintero, L., Passanha, W. D., Aubry, E., \& Poitrenaud, H. (2015). Advanced microemulsion cleaner fluid applications in deepwater wells. Offshore Technology Conference Brasil held in Rio de Janeiro, 27-29 October, Brazil. doi: 10.4043/26344-MS

Quintero, L., Salager, J-L., Forgiarini, A., Pietrangeli, \& G., Brege, J. (2012). Efficient displacement of synthentic or oil-based mud and transitional phase inversion. AlChe Spring Meeting.

Rossi, C. G. F. T. (2006). Tensoativos: uma abordagem básica e perspectivas para aplicabilidade industrial. Revista Universidade Rural, Série. Ciências Exatas e da Terra, Seropédica, V. 25(1-2), 73-85. 